\title{
Testing a Rapid Diagnostic Medium for Erwinia amylovora and Development of a Procedure for Sampling Blossoms in Pear Orchards
}

\author{
G. Kritzman, H. Shwartz, R. Marcus, S. Manulis, F. Klietman, D. Oppenheim, M. Zilberstaine, and D. Shtienberg
}

First, second, fourth, fifth, and eighth authors: Department of Plant Pathology, and third author: Department of Statistics, ARO, the Volcani Center; Bet Dagan 50250, Israel; and sixth and seventh authors: Extension Service, Ministry of Agriculture, Bet Dagan 50250, Israel. Accepted for publication 28 February 2003.

\begin{abstract}
Kritzman, G., Shwartz, H., Marcus, R., Manulis, S., Klietman, F., Oppenheim, D., Zilberstaine, M., and Shtienberg, D. 2003. Testing a rapid diagnostic medium for Erwinia amylovora and development of a procedure for sampling blossoms in pear orchards. Phytopathology 93:931-940.

The coliform agar produced by Merck was tested for rapid diagnosis of Erwinia amylovora (the causal agent of fire blight) in pear blossoms. The medium enabled the diagnosis to be completed within $36 \mathrm{~h}$. Diagnoses performed with the medium were confirmed by the BIOLOG and the fatty-acid profile methods. The diagnostic medium was used to determine the spatial distribution of colonized blossoms in the orchards and it was found that E. amylovora may be distributed both in clusters and at random. These findings were used in the development of a statistical

dence of blossoms in the orchard to be estimated at desired levels of accuracy and confidence. Parameters included in the model are: the total number of trees in the orchard $(T)$, the number of trees to be sampled in the orchard $(t)$, the number of blossoms to be sampled from each tree $(n)$, the true colonization incidence of blossoms $(\pi)$, a coefficient of aggregation $(\rho)$, the required level of confidence $(1-\alpha)$, and the required level of accuracy $(L)$. Sensitivity analyses revealed that the parameter governing sample size is the required level of accuracy. Sampling of 20 blossoms from each of several hundred trees is required to achieve an accuracy of $\pm 1 \%$, but only a few single trees are needed for an accuracy level of $\pm 10 \%$. A sampling procedure then was developed, validated with an independent data set, and found to be accurate. It was concluded that sampling of pear blossoms and estimation of the incidence of blossom colonization by $E$. amylovora could improve fire blight management, but not in all cases.
\end{abstract} model for sampling blossoms in the orchard. The model determines the number of trees to be sampled in the orchard and the number of blossoms be taken from each tree, which would enable the true colonization inci-

Fire blight, caused by the bacterium Erwinia amylovora (Burrill) Winslow et al., is the most destructive pathogen of pear and other pome-fruit trees worldwide $(15,36,39,40)$. The pathogen infects all plant parts, including blossoms, fruit, leaves, shoots, limbs, and trunks. The effects of the disease are devastating, and severely infected trees may eventually die. Prevention of blossom infection by $E$. amylovora is the key to fire blight management. Prior to infection, populations of E. amylovora can expand their size greatly through an epiphytic phase that occurs on floral surfaces. Stigmas have been shown to be the primary sites of epiphytic colonization by E. amylovora and most recent studies support the hypothesis that blossom blight is the result of initial colonization of stigmatic surfaces by E. amylovora, followed by the external washing of cells from the stigma to the hypanthium, where infection occurs (15). On the hypanthium, E. amylovora gains entry into the plant through nectarthodes located on the hypanthial surface (36). Therefore, rain or heavy dew at the end of a warm period promotes the infection (15).

Blossom infection may be prevented by application of bactericides. Blossoms, which are the most susceptible host tissue, are opened daily during bloom, and there are no effective systemic bactericides; therefore, blossoms that open after spraying are not protected. Consequently, for optimal chemical protection, sprays should be applied frequently, with short intervals between sprayings. This is not practicable because of the cost of spraying and

Corresponding author: D. Shtienberg; E-mail address: danish@ volcani.agri.gov.il

Publication no. P-2003-0603-01R

(C) 2003 The American Phytopathological Society
Additional keywords: diagnosis, integrated pest management, sampling model.

because of environmental considerations. Moreover, to lower the probability that the pathogen population will develop resistance to the bactericides, spraying should be minimized. The solution is to apply the bactericides only when necessary, according to a warning system. For example, Billing, working in southeast England, developed a system called BIS95 (3), MARYBLYT was developed by Steiner in Maryland (35), Cougarblight was developed by Smith in the Pacific Northwest (33), and the Fire Blight Control Advisory system (FBCA) was developed by Shtienberg and coworkers in Israel (31).

When prevention of blossom infection is attempted, only the environmental component of the disease triangle is considered; the host component is not considered because blossom infections are likely only during bloom when the host is always receptive to infection, and the pathogen component is not considered because it is not yet possible to determine soon enough whether the blossoms are colonized with E. amylovora. Although attempts have been made in some warning systems to estimate the likelihood of inoculum presence in the orchard $(31,33)$, the underlying assumption in all systems is that the blossoms are colonized with the pathogen. This assumption is incorrect in at least some of the cases and may lead to unnecessary spraying. The situation could be improved if reliable data on the blossom colonization situation were available on time and, because the extent of colonization may change rapidly, such data should present the situation at, or shortly before, the time of bactericide spraying. Accordingly, common sense indicates that information on the blossom colonization situation should be obtained at the farm level, preferably independently by the decision maker. For that purpose, a diagnostic tool is required. 
Various tools have been developed for identification of the causative agent, the gram-negative bacterium E. amylovora $(1,2)$. Among them are semiselective agar plates, serological assays such as enzyme-linked immunosorbent assay (ELISA) and immunofluorescence (IF), and DNA hybridization and polymerase chain reaction (PCR) techniques. Detection by serological and molecular techniques is fast and accurate but requires equipment that is common only in laboratories. Semiselective agar plates, on the other hand, may be used on the farm level. Many semiselective media can detect $E$. amylovora $(6,9,14,18,21,26)$, but the time needed for reliable detection with these media is a few days; too long for the results of the assay to be used in decision making. Moreover, in some cases, it is not possible to distinguish easily on the medium between colonies of E. amylovora and those of other bacteria that commonly inhabit pear blossoms. Consequently, the existing semiselective media cannot be used as a diagnostic tool for E. amylovora at the farm level.

Development of a fast and reliable diagnostic tool is only the first step. At full bloom, hundreds of blossoms are opened on every pear tree; hundreds of thousands in an average-size orchard. A crucial problem is to determine how to collect blossoms so that the sample would reliably represent the population of blossoms that are opened in the orchard. There is a need to define, in advance, how to choose the trees (within the orchard) and the blossoms (within each tree) to be sampled, and how many trees and blossoms to sample. Sampling procedures for determining the incidence of blossom colonization with $E$. amylovora have not yet been developed, but such procedures have been developed for other pathogens in other hosts. For example, sampling procedures were developed for Septoria tritici blotch, powdery mildew, yellow rust, foot rot, and Rhizoctonia root rot in winter wheat (27); stripe rust in wheat (10); Phomopsis leaf blight (37) and leaf spot (38) in strawberry; and downy mildew in grape (24) and cabbage (34).

For development of a sampling procedure, quantitative information is required on the spatial distribution of the pathogen or the disease in the orchard. However, to the best of our knowledge, such information on E. amylovora in pear is not available in the literature. Statistical methods, such as geostatistics, can be used to determine the spatial distribution of pathogens $(13,17)$ and this technique has been used in plant pathology to quantitatively characterize changes in the patterns of disease over time and space $(7,20,28,34)$. A curve representing changes in the spatial dependency and variability over a distance is called a semivariogram. The shape of a semivariogram may take on several different forms. For example, when the records are spatially dependent, the semivariogram exhibits a saturation curve which rises with increasing distance between sample locations until it approaches a constant values (still) at a given separation distance (range). Samples separated by a distance less than this range are spatially related, but those that are further apart are spatially independent. Conversely, when records are spatially independent, values of the semivariogram do not change with distance and the semivariogram approximates a horizontal line $(7,20,28)$.

The objectives of the present study were to (i) test a rapid diagnostic medium for determining if pear blossoms are colonized by E. amylovora, (ii) characterize the spatial distribution of blossoms colonized with E. amylovora or infected by fire blight in pear orchards, and (iii) develop a procedure for sampling pear blossoms and use this procedure for estimating E. amylovora colonization incidence.

\section{MATERIALS AND METHODS}

Testing of a rapid diagnostic tool. The coliform agar medium produced by Merck was tested for rapid diagnosis (RD-medium) of E. amylovora in pear blossoms. The composition of the medium (grams per liter of distilled water) is as follows: peptone,
3.0; sodium chloride, 5.0; sodium dihydrogenophosphate, 2.2; disodium hydrogenphosphate, 2.7 ; tryptophan, 1.0; sodium pyruvate, 1.0; tergitol 7, 0.15; sorbitol, 2.0; Chromogenic mix 0.4; and agar, 10.0. Benomyl (50\% a.i.), $50 \mathrm{mg}$, or cycloheximide (Sigma), $50 \mathrm{mg}$, were added to the medium to inhibit the development of fungi.

The accuracy of the diagnosis attained with the RD-medium was tested as follows. Blossoms were randomly sampled from four pear orchards located in northern Israel; 120 trees were sampled in each orchard and 10 blossoms were taken randomly from each tree. The blossoms were imprinted onto petri dishes into which the RD-medium had been poured. Randomly selected colonies that exhibited the typical color and shape of E. amylovora on the RD-medium, as well as colonies that did not exhibit them, were sent for identification by means of two common methods for diagnosis of bacteria (i.e., the BIOLOG method and the fatty-acid profile method). The BIOLOG microplate system provides accurate identification of the genus, species, and pathovar of phytopathogenic bacteria $(4,5)$, including $E$. amylovora (30); it is based on the reactions of isolates of the tested bacteria to a series of 95 carbon sources. Positive use of a carbon source by the isolate is indicated by a color reaction and this is called the organism's "metabolic fingerprint" (16). Microbial identification based on the fatty-acid profile is one of the stable methods. The tested bacteria are grown under standard conditions (media, temperature, and time); they then are harvested and their fattyacid contents are purified and injected into high-performance liquid chromatography. Identification is done by comparing the fatty-acid profile of the tested bacteria with that of various bacterial species that are stored in a computer database of the MIDI Sherlock Mycobacteria Identification System (MIDI Inc., Newark, $\mathrm{DE})$. Results are given as percentage similarity of the fatty-acid profile to those of known bacteria in the database (29).

The speed with which colonies could be identified as E. amylovora by means of the RD-medium was tested in two experiments. Ten isolates previously identified as E. amylovora by both the BIOLOG and the fatty acid-profile methods were plated onto the RD-medium in $1-\mu l$ drops $\left(10^{6}\right.$ E. amylovora cells $\left./ \mathrm{ml}\right)$. The plates were incubated at room temperature $\left(\approx 20\right.$ to $\left.25^{\circ} \mathrm{C}\right)$ to simulate situations where temperature-controlled incubators are not available. The plates were inspected periodically, starting after $10 \mathrm{~h}$ of incubation, and colonies that exhibited the typical color and shape of E. amylovora on the plates were marked. The cumulative proportion of colonies identified as E. amylovora was plotted over time to determine the speed of identification.

The spatial distribution of $E$. amylovora in pear orchards. The spatial distributions of blossoms colonized with $E$. amylovora and of blossom clusters exhibiting fire blight symptoms were determined in pear orchards in 1999 and 2000. The orchards were not treated with bactericides and the blossoms were naturally colonized or infected with the pathogen. In 1999, fire blight severity was assessed in two orchards, one located in northern Israel and the other in the coastal plain. In the first orchard, 50 trees in a row were assessed; in the second orchard, three rows (each of 50 trees) were assessed. Two individuals inspected the trees from both sides, and counted the number of infected blossom clusters per tree (i.e., a measure of disease severity). In 2000, the incidence of blossom colonization with E. amylovora was determined with the RD-medium. Blossoms were sampled from two rows of pear trees (each of 30 to 50 trees) in two orchards located in the coastal plain. Sampling was performed three times during bloom: when $5 \%$ of the blossoms were open, when 40 to $50 \%$ of the blossoms were open ( 1 week later), and at full bloom (5 days later). In all, 20 blossoms were randomly sampled from each tree and imprinted onto the RD-medium, 10 blossoms per plate. The plates were incubated at room temperature for $36 \mathrm{~h}$, after which the incidence of blossom colonization was determined visually. The geostatistical software GS+ (version 2.3; Gamma Design 
Software, Plainwell, MI) was used to determine the spatial dependency of E. amylovora colonization (in 2000) and of fire blight infections (in 1999) in the orchards. The spatial distribution of the pathogen in the orchards was determined from the shape of the resulting semivariogram.

Development of a procedure for sampling pear blossoms. The goal of the sampling procedure developed in the present study was to estimate the true colonization incidence of blossoms with a required level of accuracy, at a required level of confidence. The sampling procedure was developed in several steps, as follows. First, a theoretical statistical sampling model was assumed. Parameters used in the model were the total number of trees in the orchard $(T)$, the number of trees to be sampled in the orchard $(t)$, the number of blossoms to be sampled from each tree $(n)$, the true colonization incidence of blossoms $(\pi)$, a coefficient of aggregation $(\rho)$, the required level of confidence $(1-\alpha)$; and the required level of accuracy $(L)$. Basic steps in the development and the assumptions underlying the model are elaborated below. The model then was used for theoretical calculations in which a range of values was assigned to each of the model's parameters (sensitivity analyses). These tests enabled the relative influence of each parameter on sample size to be determined. Finally, calculations based on the relationship between $\rho$ and $\pi$, as was established quantitatively by data recorded in the pear orchards in 2000, were used to develop a practical sampling procedure for pear blossoms.

The sampling procedure was evaluated with data recorded in commercial orchards in 2002, and its ability to provide the required levels of accuracy and confidence was tested. Twenty pear blossoms were sampled from each of 25 pear trees in two commercial orchards that had not been treated with bactericides. The $E$. amylovora colonization incidence was determined with the RD-medium. The mean blossom colonization incidence of the 25 trees was considered as the true colonization incidence of blossoms. As will be elaborated below, according to the developed sampling procedure, sampling of 20 blossoms from each of four trees would enable the true colonization incidence to be determined with an accuracy of $\pm 10 \%$ in confidence of $90 \%$. One hundred samples of four trees were randomly chosen from the data set of the 25 trees and an average colonization incidence was calculated for each sample. The deviation of the sample average from the true colonization incidence was calculated for each sample. The frequency distribution around zero of the deviations for the 100 samples (i.e., the true colonization incidence) was plotted, and used to determine whether the required levels of accuracy and confidence had been attained.

Statistical sampling model. General background. Consider an orchard with $T$ trees. We first assume that $T$ is very large (theoretically, $T=\infty$ ). Suppose that $t$ trees are randomly sampled from the orchard and $n$ blossoms are drawn from each tree and tested for colonization with E. amylovora. Let $X_{i}$ represent the observed number of colonized blossoms out of $n$ blossoms drawn from the $i$ th tree $(i=1, \ldots, t)$. The data consist of $t$ observations $X_{i}$, $i=1, \ldots, t$, or equivalently observed proportions of colonized blossoms per tree $X_{\mathrm{i}} / n i=1, \ldots, t$. When there is a random pattern of colonized blossoms over the orchard, it can be assumed that the blossoms sampled from the orchard form $N=n t$ "independent Bernoulli trials" with a common probability of a blossom being colonized, say $p$. In this case, an appropriate distribution of $X_{i}$ (or $\left.\Sigma X_{i}\right)$ is the binomial distribution $B(p, n)$ (or $B(p, n t)$ ). However, if colonization probabilities vary among trees in the orchard, the binomial distribution is not appropriate. Statistical methods for modeling variability in this type of data are described, for example, by Collett (8). It is shown that under the following conditions (i) the colonization probability for the $i$ th tree, say $p_{i}$, is an unobservable random variable with mean $\pi$ and variance $V$ given by $V=\varphi \pi(1-\pi)$, where $\varphi(\geq 0)$ is an unknown scale parameter; and (ii) the conditional distribution of $X_{i}$, given a particular value of $p_{i}$, is binomial $B\left(p_{i}, n\right)$. It follows that the mean and variance of $X_{i}$ are given by

$$
\begin{gathered}
E\left(X_{i}\right)=n \pi \\
\operatorname{Var}\left(X_{i}\right)=n \pi(1-\pi) \eta
\end{gathered}
$$

where

$$
\eta=1+\varphi(n-1)
$$

Usually, the parameter $\varphi$ is restricted to be less than 1 . The parameter $\pi$ represents the mean colonization probability or true colonization incidence. The parameter $\eta$ is known as the heterogeneity or the overdispersion parameter. When colonization probabilities vary among trees in the orchard, the data are said to exhibit extrabinomial variation or overdispersion. In this case, $\eta>$ 1 and the variance of $X_{i}$ (or $\Sigma X_{i}$ ) is greater than the corresponding binomial variance, which is given by $n \pi(1-\pi)$ (or $n t \pi(1-\pi)$ ), by the factor of $\eta$. If there is no variation among trees $(\eta=1)$, the distribution of each $X_{i}$ reduces to $B(\pi, n)$.

A more specific assumption about the variability of $p_{i}$ is based on the parametric form of the distribution of $p_{i}$, which can be modeled by Beta distribution $\beta(a, b)$ with $a \geq 0, b \geq 0$. For this distribution,

$$
\begin{aligned}
& E(p)=a /(a+b) \equiv \pi \\
& \operatorname{Var}(p)=\rho \pi(1-\pi)
\end{aligned}
$$

where $\rho=1 /(a+b+1)(0 \leq \rho \leq 1)$. In this case, the distribution of $X_{i}$ is beta-binomial $B B(\pi, n, \rho)$ with mean given by equation 1 and variance given by equation 2 with $\varphi=\rho$. Parameter $\rho$ sometimes is called the aggregation parameter or intraclass (intracluster) correlation. When $\rho=0(a+b \rightarrow \infty)$, the pattern of colonized blossoms is random over the orchard and the distribution of $X_{i}$ reduces to $B(\pi, n)$. When $\rho>0$, the colonization probability varies among the trees, indicating clusters of colonized blossoms in the orchard. It often is assumed that both parameters $a$ and $b$ are greater than 1 (the distribution of $\rho$ is unimodal) and, hence, $0 \leq$ $\rho \leq 1 / 3$.

Estimation of $\pi$ and $\eta$. When overdispersion (extrabinomial variation) is being modeled, the moment estimates of $\pi$ and $\eta$ are given by

$$
\begin{aligned}
& \hat{\pi}=\sum_{i=1}^{t} X_{i} / t \\
& \hat{\eta}=s_{\text {obs }}^{2} / s_{\text {bin }}^{2}
\end{aligned}
$$

where the estimates $s_{\text {obs }}^{2}$ and $s_{\text {bin }}^{2}$ are defined by

$$
\begin{gathered}
s_{\mathrm{obs}}^{2}=\sum_{i=1}^{t}\left(X_{i} / n-\hat{\pi}\right)^{2}(t-1) \\
s_{\text {bin }}^{2}=\hat{\pi}(1-\hat{\pi}) / n
\end{gathered}
$$

Thus, $\hat{\pi}$ is the observed proportion of colonized blossoms in the orchard and $\eta$ is the ratio of the observed variance of colonized blossoms sampled from a tree $\left(s_{\text {obs }}^{2}\right)$ to the variance should the proportion have a binomial distribution $\left(s_{\text {bin }}^{2}\right)$. Using the relation $\eta=1+(n-1) \rho$, the parameter $\rho$ may be estimated by:

$$
\hat{\rho}=(\hat{\eta}-1) /(n-1)
$$

When the data $X_{i}(i=1, \ldots, t)$ fit the beta-binomial distribution $B B(\pi, n, \rho)$, the moment estimates $\pi$ and $\rho$ given in equations 6 and 10 can be used as model estimates. Alternatively, maximum likelihood estimation procedure may be used for obtaining model parameter estimates and its standard errors.

Modeling plant disease incidence data are widely used by plant epidemiologists. The beta-binomial distribution was shown to fit incidence of diseases caused by viruses, fungi, and bacteria. A thorough discussion of this subject is presented by Hughes et al. (11), Madden et al. (25), Madden and Hughes (22,23), and others. 
Determination of the number of trees ( $t$ ) to be sampled. Based on the above assumptions, the variance of $\hat{\pi}$ is given by the following equation:

$$
\operatorname{var}(\hat{\pi})=\pi(1-\pi)[1+(n-1) \rho] /(n t)
$$

Equation 11 implies that when $\rho=0, \operatorname{var}(\hat{\pi})$ coincides with the variance of the binomial distribution $B(\pi, n t)$. When the sample size is large, an approximate confidence interval for the parameter $\pi$, with a confidence level of $1-\alpha$, is given by

$$
\pi \in \hat{\pi} \pm z_{1-\alpha / 2} \sqrt{\operatorname{va\hat {r}}(\hat{\pi})}
$$

where $Z_{1-\alpha / 2}$ denotes the upper percentile $(1-\alpha / 2)$ of a standard normal distribution and $\operatorname{var}(\hat{\pi})$ represents the estimated variance of $\hat{\pi}$. This estimated variance may be obtained from equation 11 by replacing $\pi$ with $\hat{\pi}$.

The number of trees to be sampled is determined by satisfying the requirement that the estimated colonization incidence $(\hat{\pi})$ should not differ from the true incidence $(\pi)$ by more than a certain value, say $L$, for a certain confidence level, $1-\alpha$. Thus, $L$ represents the accuracy of the estimation. This requirement may be expressed as

$$
\operatorname{Pr}\{|\pi-\hat{\pi}| \leq L\}=1-\alpha
$$

Clearly, increasing the required level of accuracy (i.e., decreasing the value of $L$ ) would increase the sample size. Using equation 12, equation 13 may be modified to

$$
z_{1-\alpha / 2} \sqrt{\operatorname{var}(\hat{\pi})} \leq L
$$

Inserting the term $\operatorname{var}(\hat{\pi})$ from equation 11 into equation 14 , provides the solution for the number of trees $(t)$ to be sampled that will ensure the required levels of accuracy $(L)$ for a certain level

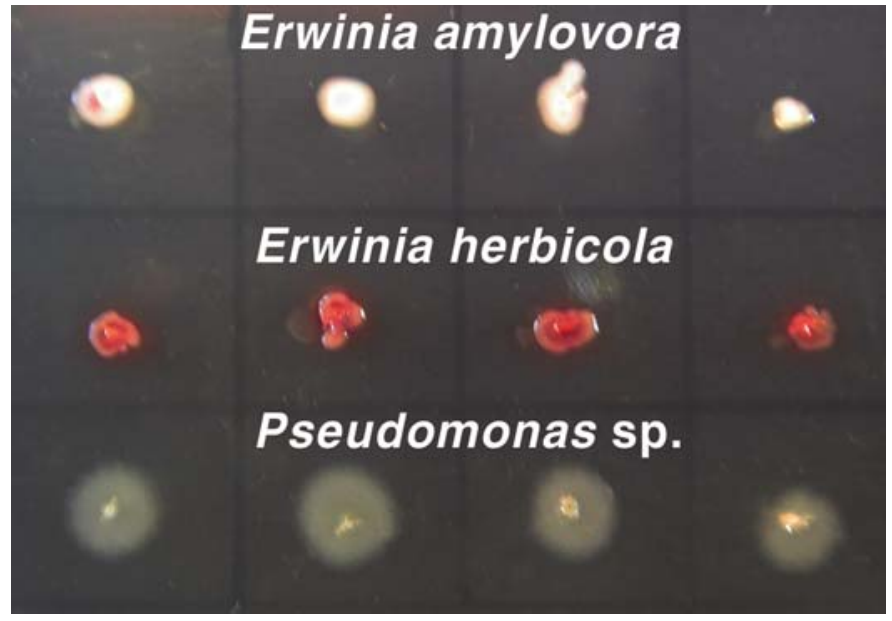

Fig. 1. Characteristic colonies of three bacterial species on the rapid diagnosis medium after $36 \mathrm{~h}$ of incubation at room temperature $\left(20\right.$ to $\left.25^{\circ} \mathrm{C}\right)$. Four different strains were plated for each species.

TABLE 1. Verification of the diagnosis of Erwinia amylovora strains done by the rapid diagnostic medium using the BIOLOG and the fatty-acid profile methods $^{\mathrm{a}}$

\begin{tabular}{lccccc}
\hline & \multicolumn{2}{c}{ BIOLOG method } & & \multicolumn{2}{c}{ Fatty-acid profile method } \\
\cline { 2 - 3 } \cline { 5 - 6 } Orchard no. & Tested & Confirmed & & Tested & Confirmed \\
\hline 1 & 17 & 17 & & 59 & 57 \\
2 & 6 & 6 & & 23 & 23 \\
3 & 9 & 9 & & 19 & 19 \\
4 & 9 & 9 & & 26 & 25 \\
Total & 41 & 41 & & 127 & 124 \\
\hline
\end{tabular}

${ }^{\text {a }}$ Tested $=$ number of tested isolates. Confirmed $=$ number of isolates confirmed to be E. amylovora. of confidence $(1-\alpha)$ for an orchard with large number of trees $(T \cong \infty)$. It is given by

$$
t=\left(Z_{1-\alpha / 2}\right)^{2} \pi(1-\pi)(1+[n-1] \rho) /\left(n L^{2}\right)
$$

Note that $\operatorname{var}(\hat{\pi})$ and hence $t$ depend on the true colonization incidence $\pi$ and the coefficient of aggregation, $\rho$, which are unknown parameters. Equation 15 is the same as equation 13 of Hughes et al. (11), who considered cluster sampling procedure for disease incidence data with a preassigned accuracy level defined by equation 14. These authors also provided formulae for two other accuracy level definitions.

When the number of trees in the orchard $(T)$ is finite, the variance of $\hat{\pi}$ is given by the equation:

$$
\operatorname{var}(\hat{\pi})=\pi(1-\pi)(1+[n(1-t / T)-1] \rho) /(n t)
$$

Similarly, substituting the term $\operatorname{var}(\hat{\pi})$ from equation 16 in equation 14 results in a formula for the number of trees $(t)$ to be sampled that fulfills the required levels of accuracy $(L)$ and confidence $(1-\alpha)$, for an orchard with a finite number of trees $(T)$ :

$$
t=\frac{\pi(1-\pi)[1+\rho(n-1)]}{n\left[L^{2} /\left(z_{1-\alpha / 2}\right)^{2}+\pi(1-\pi) \rho / T\right]}
$$

When the number of trees in the orchard is large enough, the correction factor $\pi(1-\pi) \rho / T$ in equation 17 is negligible, and equation 15 may be used.

In summary, when overdispersion for blossom colonization is modeled, equation 15 can be used to determine the number of trees $(t)$ to be sampled from pear orchards with a large number of trees. Parameters included in equation 15 are the number of blossoms to be sampled from each tree $(n)$, the coefficient of aggregation $(\rho)$, the true colonization incidence of the blossoms $(\pi)$, and the required levels of accuracy $(L)$ and confidence $(1-\alpha)$. Incorporating reasonable values of $\pi, \rho$, and $L$ and various values of $n$ in equation 15 results in the corresponding values of trees $(t)$ to be sampled. The true colonization incidence of the blossoms $(\pi)$ is not known before sampling; therefore, the sampling procedure developed in this study assumed the largest possible value of $\operatorname{var}(\hat{\pi})$ which was attained at $\pi=50 \%$.

It may be expected that the proportions of colonized blossoms per tree show some aggregations within a branch or even a blossom cluster in each tree. Such aggregation on spatial hierarchy may affect the sampling scheme (12). It can be shown that, when the true colonization incidences vary among branches within trees and among trees in the orchard, the variance of $X_{i}$ will depend on the following parameters: true mean colonization incidence $(\pi)$, heterogeneity of branches within a tree, and aggregation caused by variation among trees. With a cluster sampling of $t$ trees and $n$ blossoms per tree, as with our data, it is not possible to obtain separate estimates for these two sources of aggregations. In order

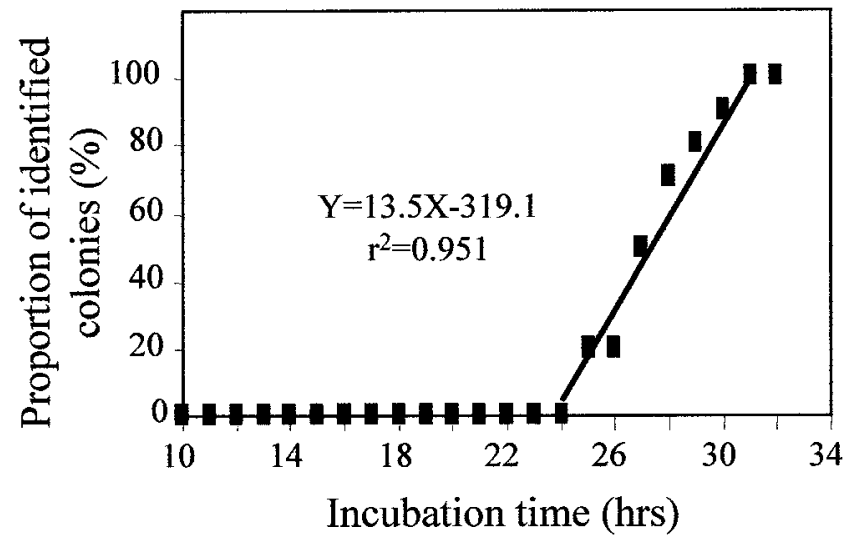

Fig. 2. Proportion of colonies identified as Erwinia amylovora on rapid diagnosis medium as a function of the incubation time. 
to get direct estimates to two aggregation parameters, one should take a sample of $t$ trees, $k$ branches from each tree, and $n$ blossoms from each branch and observe the number of colonized blossoms out of $n$ blossoms sampled from a branch per tree.

\section{RESULTS}

Testing of a rapid diagnostic tool. E. amylovora colonies could be distinguished easily from colonies of other bacteria and fungi that grew on the RD-medium. E. amylovora colonies had a typical white, domed, shining, mucoid appearance with radial striations and with a dense flocculent center or central ring that turned out to be reddish within $23 \mathrm{~h}$. For illustration, pure cultures of strains identified as E. amylovora, and of E. herbicola and Pseudomonas spp. (which commonly inhabit pear blossoms), were plated onto the RD-medium. The developed colonies of the three bacteria species differed in color and shape after $36 \mathrm{~h}$ of incubation at room temperature (Fig. 1).

Pear blossoms that appeared visually to be noninfected, as well as blossoms that exhibited the typical fire blight symptoms, were used to verify the accuracy of the RD-medium. The blossoms were imprinted onto the RD-medium and colonies with various colors and shapes grew within 25 to $40 \mathrm{~h}$ of incubation at room temperature. The addition of benomil or cycloheximide to the medium precluded the development of most microorganisms that commonly inhabit pear blossoms and which are abundantly present in pear orchards. Thus, keeping the petri plates open while imprinting the blossoms onto the RD-medium did not result in excessive contamination of the plates. Of the isolates identified as
E. amylovora on the RD-medium, 41 were randomly selected for confirmation with the BIOLOG method. The visual identifications done based on the RD-medium were confirmed by the BIOLOG method in all cases (Table 1). Similarly, 127 isolates randomly selected from the collection were identified according to their fattyacid profiles and 124 of them $(97.6 \%)$ were confirmed to be $E$. amylovora (Table 1). The pathogenicity of representative isolates identified as E. amylovora was tested by stabbing green pear fruits. All were proved to be pathogenic. For estimation of possible erroneous negative identification, 12 isolates that were not suspected to be E. amylovora (as determined on the RDmedium) were sent for identification by the BIOLOG and the fatty-acid profile methods. All nonsuspected strains were confirmed to be microorganisms other than E. amylovora; among them were E. herbicola, Pseudomonas spp., and yeasts. The recovery rate of $E$. amylovora populations by the RD-medium was comparable $(98.3 \pm 0.32 \%)$ with that achieved by nutrient agar; both media were able to recover less than $10 \mathrm{E}$. amylovora cells (data not shown).

Information on whether the blossoms are colonized with $E$. amylovora would be relevant for decision making only if it was obtained within less than $48 \mathrm{~h}$ after sampling. Bacteria isolates previously identified as E. amylovora were plated onto the RDmedium and the plates were kept at room temperature. After $25 \mathrm{~h}$ of incubation, typical reddish-colored colonies were apparent on $20 \%$ of the platings. Within an additional $5 \mathrm{~h}, 90 \%$ of the colonies exhibited the characteristic appearance and, $1 \mathrm{~h}$ later (i.e., after $31 \mathrm{~h}$ of incubation), all colonies could be identified easily as $E$. amylovora (Fig. 2). In a second experiment, all colonies exhibited
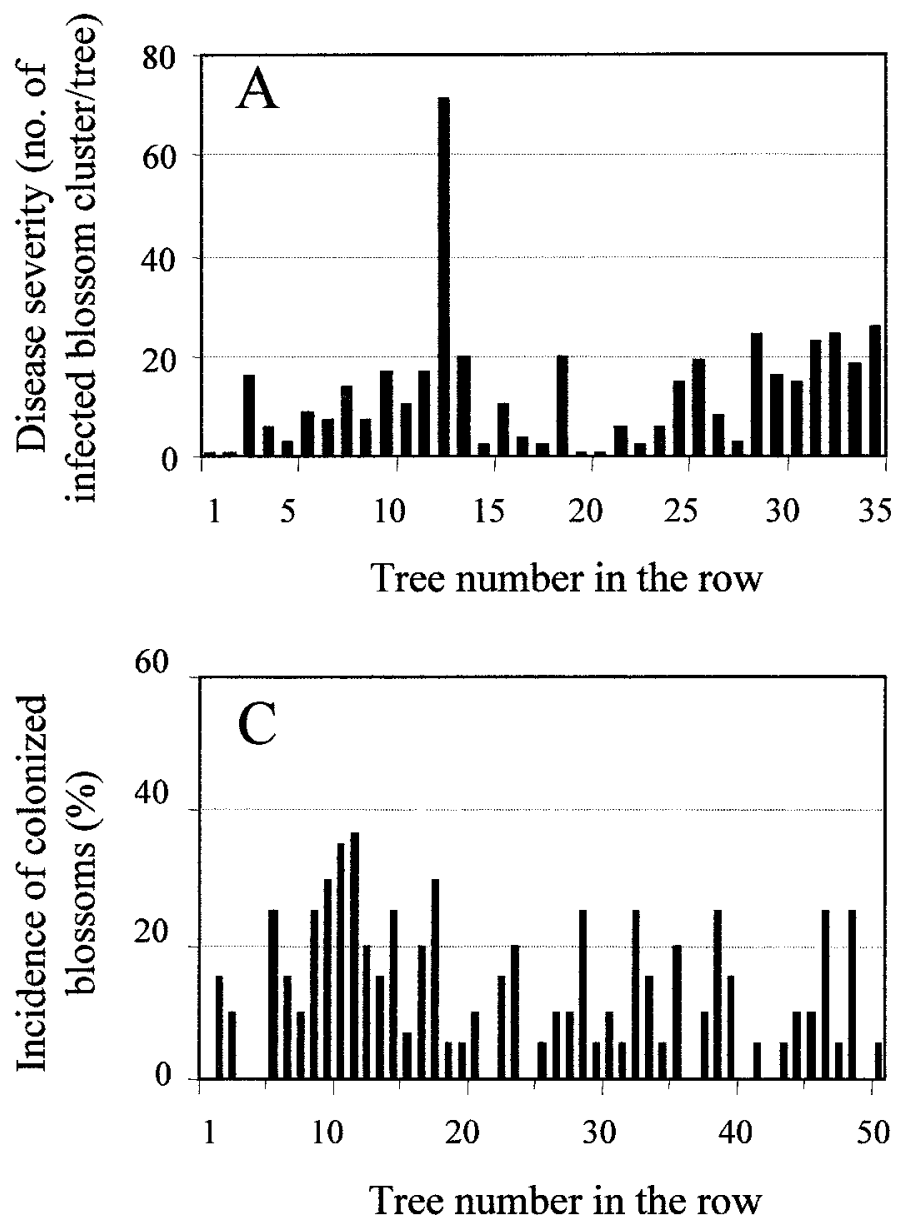
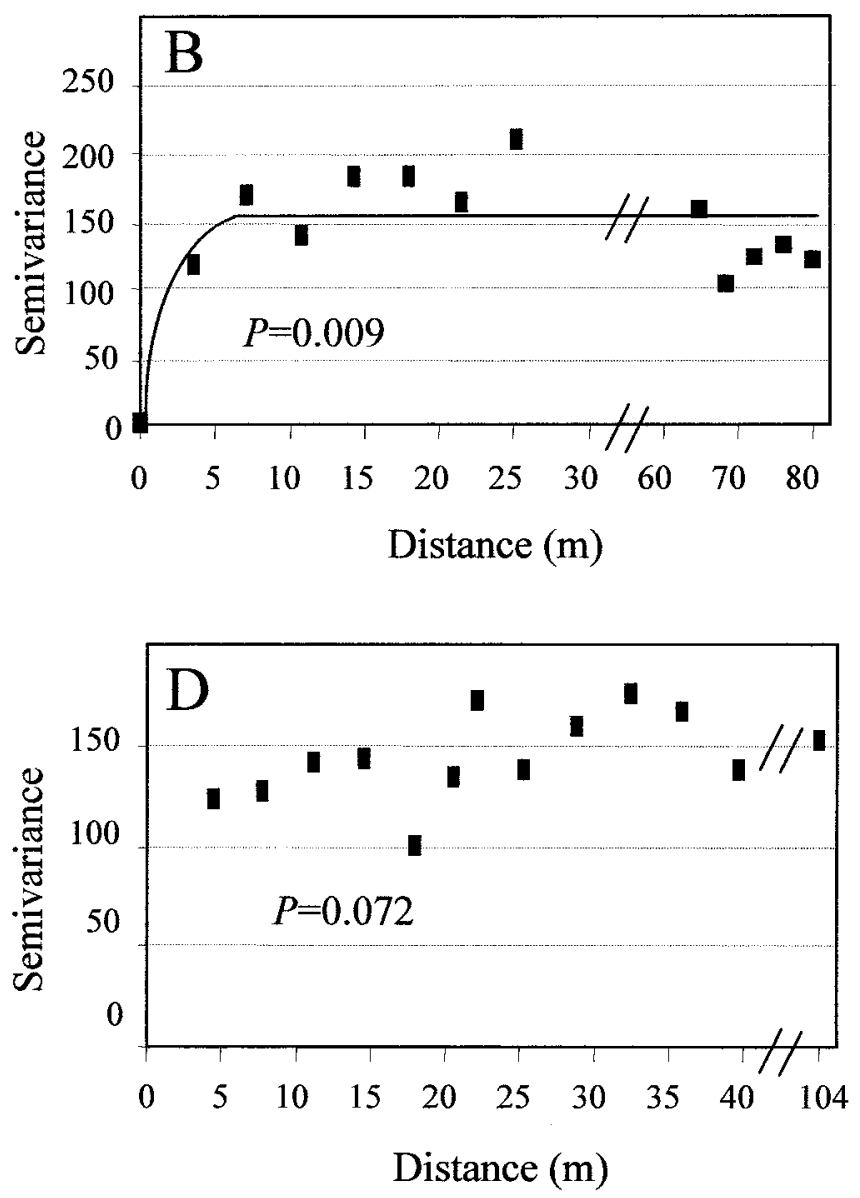

Fig. 3. Spatial distribution of fire blight (caused by Erwinia amylovora) in a row of pear trees. A, Number of infected blossom clusters and $\mathbf{C}$, the incidence of colonized blossoms per tree. B and D, Semivariograms illustrating the spatial dependency between trees in the row, as determined by geostatistical analysis. Data were recorded in orchards that were not sprayed with bactericides. 
the characteristic appearance of E. amylovora within $36 \mathrm{~h}$ of incubation at room temperature.

The spatial distribution of $E$. amylovora in pear orchards. The spatial distributions of E. amylovora-colonized blossoms and of fire blight infections were determined in 1999 and 2000 in four commercial orchards that had not been treated with bactericides. The results obtained in two of the orchards are presented in this

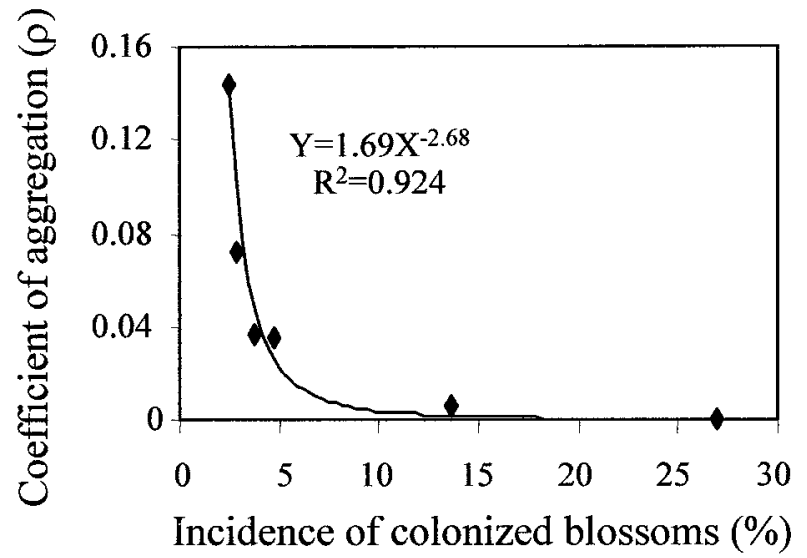

Fig. 4. Relationship between the incidence of blossom colonization by Erwinia amylovora and the coefficient of aggregation $(\rho)$. Blossoms were sampled in 1999 and 2000 from commercial orchards that were not treated with bactericides and were naturally colonized with the bacteria. Each data point represents 600 to 1,000 blossoms, sampled from 30 to 50 pear trees. The coefficient of aggregation $(\rho)$ was estimated according to equation 10 . article; those recorded in the other orchards (for both colonization and infection) had similar features. There was a large variability in the number of fire blight-infected blossom clusters (Fig. 3A) or the incidence of colonized blossoms (Fig. 3C) between trees. Geostatistical analysis of the spatial distribution of the pathogen and the disease resulted in a significant spatial dependency between infections in one case but insignificant dependency in the other (Fig. 3B and D). This implies that infections in one orchard were distributed in clusters whereas, in the other, the distribution was at random. Geostatistical analysis revealed that the spatial distribution of blossom colonization in one of the other orchards was random and that the distribution of fire blight-infected blossom clusters in the second orchard was clustered (data not shown).

Estimated overdispersion parameter of pear blossoms colonization data. Our data consist of percentage of blossoms colonized with E. amylovora out of $n=20$ blossoms drawn from a tree, where $t$ trees were sampled in commercial orchards. Values of estimated overdispersion parameter $(\hat{\eta})$ and the corresponding aggregation parameter ( $\hat{\rho})$ were calculated using equations 7 and 10 and are presented in Figure 4. When the proportion of colonized blossoms $\pi$ was low, the value of $\rho$ was high, suggesting that the colonized blossoms are not randomly distributed over the orchard, but in clusters (trees). As the colonization incidence increased, the estimated value of $\rho$ decreased progressively. At blossom colonization proportion $\pi \geq 15 \%$, the value of $\rho$ tends to zero, indicating that the colonized blossoms were randomly distributed in the orchard. Asymmetric form of the binary power law model (22) was used to relate $\rho$ to $\pi$. The fitted curve is presented in Figure 4.

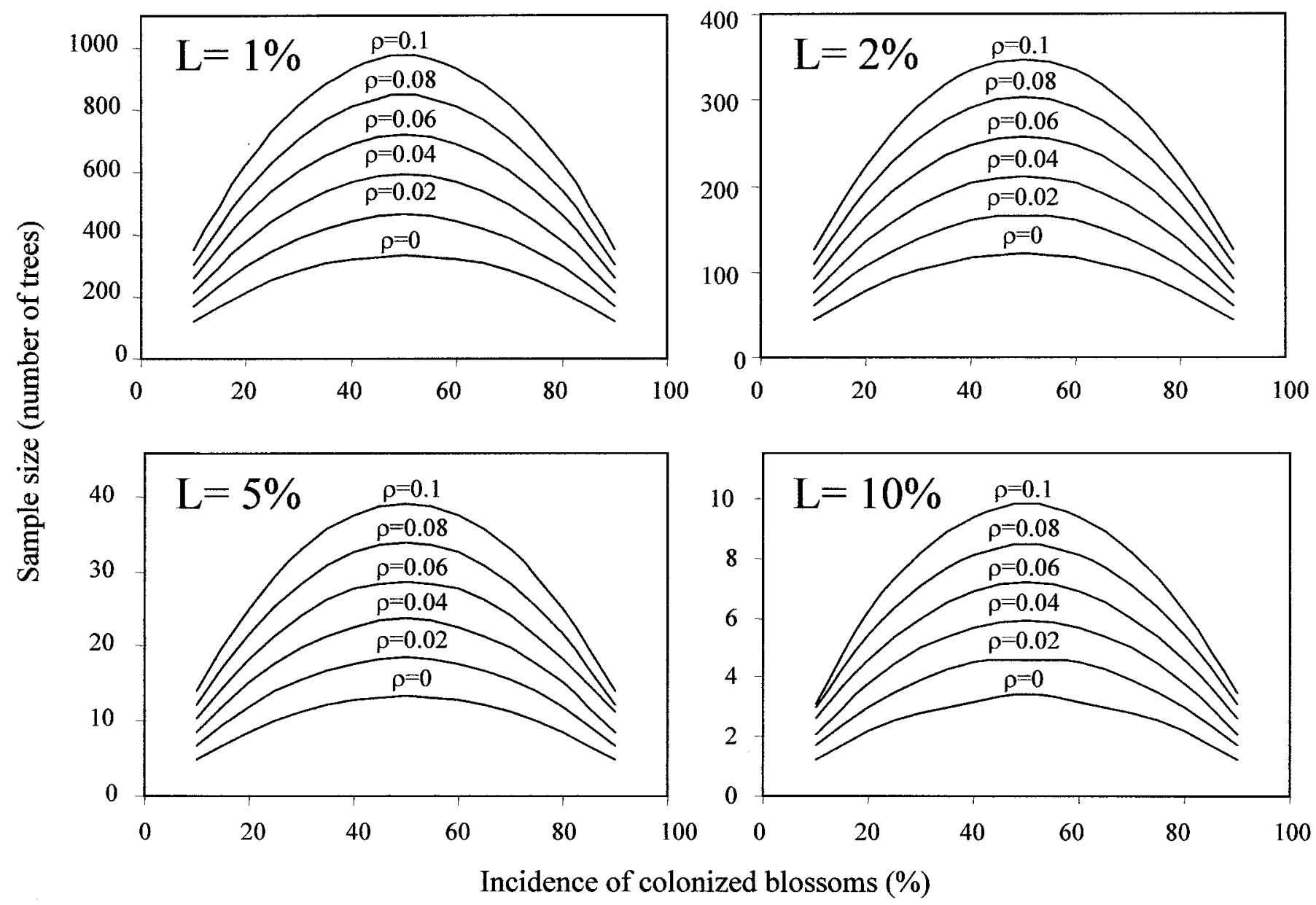

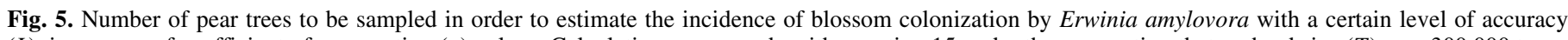

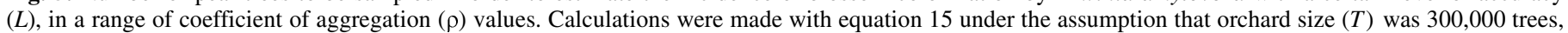
the number of blossoms sampled from each tree $(n)$ was 20 ; and the confidence level $(1-\alpha)$ was $90 \%$. 
Sensitivity analyses of the model parameters. Equation 15 was used to conduct sensitivity tests in which certain fixed values were assigned to some of the parameters and ranges were assigned to others. For example, in the calculations presented in Figure 5, the fixed parameters were orchard size $(T=300,000$ trees $)$, the number of blossoms sampled from each tree $(n=20)$, and the required level of confidence $(1-\alpha=90 \%)$. The parameters' assigned ranges were the incidence of blossom colonization with $E$. amylovora ( $\pi$, ranging from 10 to $90 \%$ ), the coefficient of aggregation ( $\rho$, ranging from 0 [random pattern of colonized blossoms] to 0.1 [clustered pattern]), and the level of accuracy ( $L$, ranging from 1 to $10 \%$ ). Solving equation 15 enabled us to determine the number of trees to be sampled (Fig. 5; $y$ axis) for each combination of parameters, so as to provide the required levels of accuracy and confidence.

Usually, sample size is related to the variability of the measured variable. When the units used for measuring the variable are proportions or percentages (such as the units of $\pi$ ), then the widest variability (and the largest sample size needed) occurs at 0.5 or $50 \%$, respectively. As expected, for each combination of parameters, sample size increased gradually until the incidence of blossom colonization reached $50 \%$, and decreased progressively thereafter (Fig. 5). The spatial pattern of colonized blossoms also affected the sample size; the necessary sample size was the smallest when the colonized blossoms were randomly distributed in the orchard (at $\rho=0$ ), and it increased gradually with increasing levels of aggregation (up to a clustered pattern at $\rho=$ 0.1 ). As expected, the required level of accuracy strongly affected sample size. To provide a required accuracy of $1 \%$ (at a confidence level of $90 \%$ ), several hundred trees had to be sampled; the number of trees to be sampled diminished to a few dozen to provide an accuracy level of 5\%, and only a few single trees had to be sampled for an accuracy level of $10 \%$ (Fig. 5). Thus, for example, if the true colonization incidence is $26 \%$ and the required level accuracy is $10 \%$, solving equation 15 determines the number of trees to be sampled to ensure that the estimated colonization incidence would fall between from 16 and $36 \%$.

Another set of calculations was conducted to test whether, for certain levels of accuracy and confidence, increasing the number of blossoms sampled from each tree (and simultaneously decreasing the number of trees sampled in the orchard) would reduce the total number of blossoms to be sampled. If the spatial distribution of colonized blossoms was random $(\rho=0)$, then sampling more blossoms from fewer trees did not make any difference, because

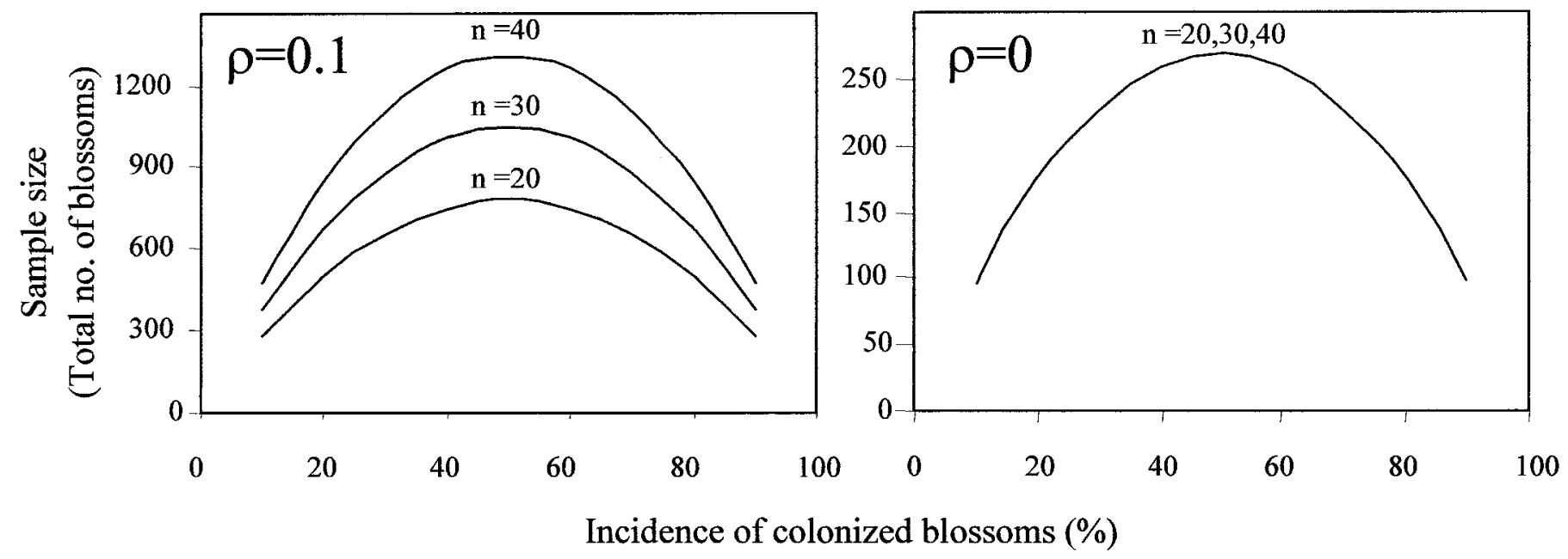

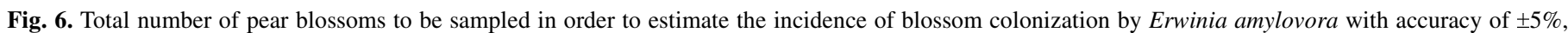

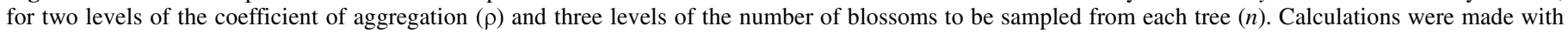
equation 15 under the assumption that the orchard size $(T)$ was 300,000 trees and the confidence level $(1-\alpha)$ was $90 \%$.

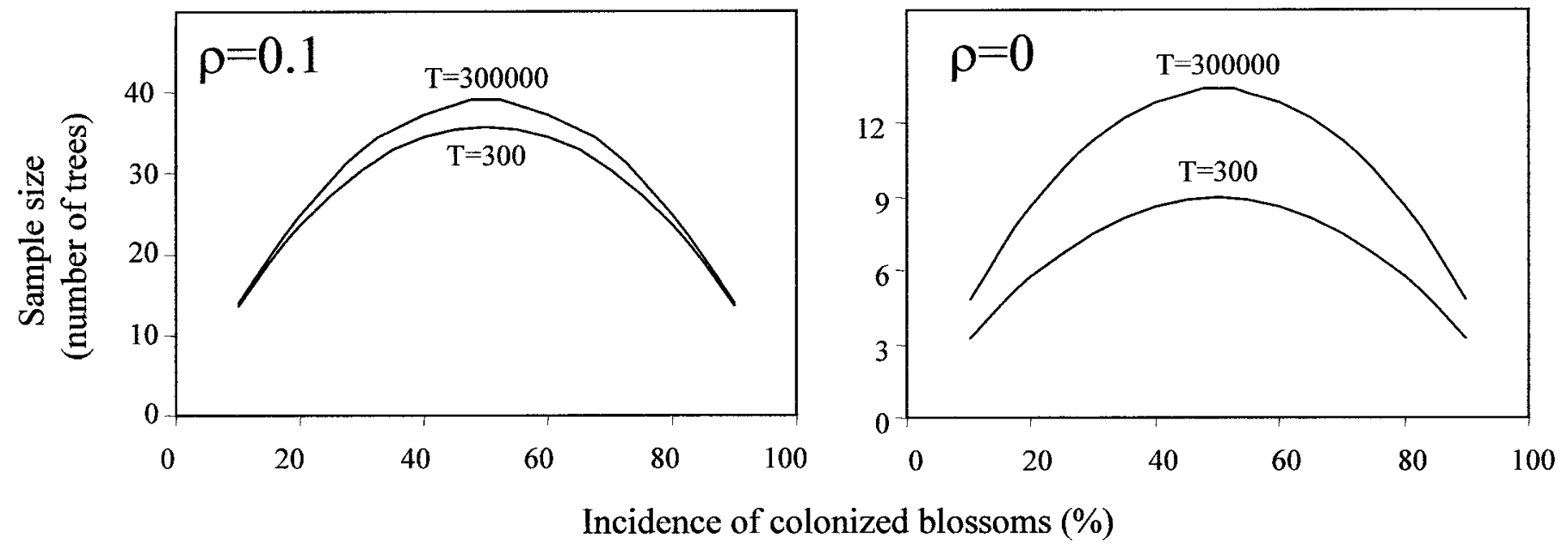

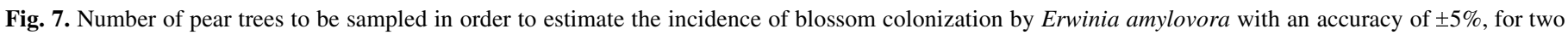

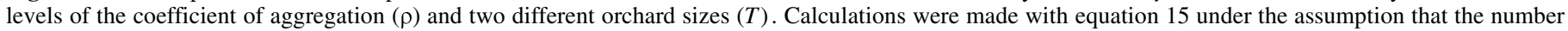
of blossoms sampled from each tree $(n)$ was 20 and the confidence level $(1-\alpha)$ was $90 \%$. 
the total number of sampled blossoms was exactly the same. However, if the colonized blossoms were distributed spatially in clusters $(\rho=0.1)$, than it would be preferable to sample fewer blossoms from more trees, than the converse (Fig. 6). Henceforth, a sample of 20 blossoms from each tree was assumed in further calculations. As expected, for any given levels of accuracy and confidence, sample size was affected by the size of the orchard $(T)$. Nevertheless, the relative influence of this parameter on sample size was minute (Fig. 7).

The relationship presented in Figure 4 implies that not all combination of the parameters $\pi$ and $\rho$ presented in Figures 5 to 7 actually occur in nature. For example, when the blossom colonization incidence is $>15 \%$, the value of $\rho$ never exceeds zero. The numbers of trees to be sampled for the combinations of $\pi$ and $\rho$ that are likely to occur in nature are presented in Figure 8.

The calculations conducted for determining the number of trees to be sampled depends on the true blossom colonization incidence; but this incidence is not known before the sampling had been done. However, as indicated above, because the units used for measuring colonization incidence are proportions, the widest variability (and, therefore, the greatest necessary sample size) for each combination of parameters occurs when the blossom colonization incidence is $50 \%$. Thus, for example, for required accuracy and confidence of 1 and $90 \%$, respectively, a sample of 20 blossoms from each of 338 trees is needed; for required accuracy and confidence of 10 and $90 \%$, respectively, a sample of 20 blossoms from each of four trees is needed (Fig. 8). If, in fact, the true incidence of blossom colonization were actually lower (or higher) than $50 \%$, then the number of sampled trees would exceed the number that was required. Nevertheless, in no case would the sample size be underestimated. The sample sizes required for various levels of accuracy and confidence are presented in Figure 9.

Sampling scheme for pear blossoms colonization. Based on the above, the following procedure for sampling blossoms was developed. (i) Define the required levels of accuracy and confidence. (ii) Determine the number of trees to be sampled, based on the relationships presented in Figure 9 (differences in sample sizes between small and very large orchards [e.g., orchards with 300 and 300,000 trees, respectively] are negligible [Fig. 7]; therefore, to avoid undersampling, the orchards could be regarded to be very large). (iii) Collect the blossoms in the orchard, choose the trees to be sampled at random, and take 20 blossoms from each tree. (iv) Imprint each blossom onto the RD-medium that had been poured into a petri plate, 10 blossoms per plate. (v) Incubate the petri plates at room temperature for $36 \mathrm{~h}$ and determine the number of

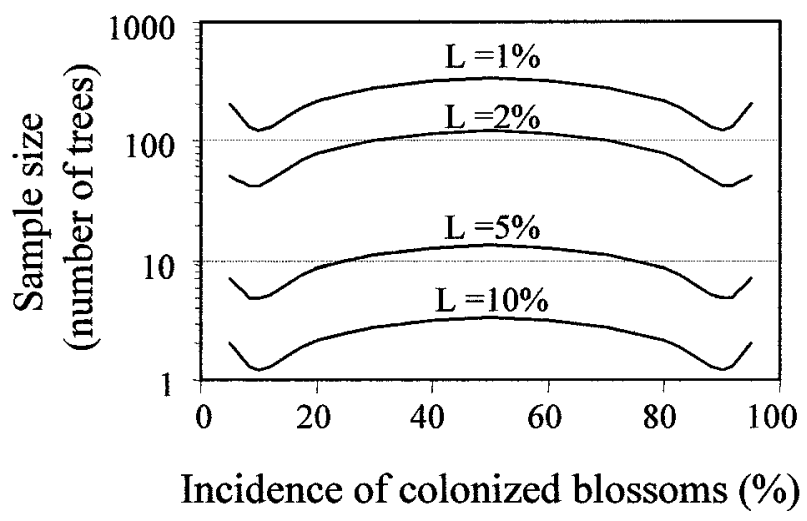

Fig. 8. Numbers of pear trees to be sampled in order to estimate the incidence of blossoms colonization by Erwinia amylovora in a range of accuracy levels $(L)$. The coefficient of aggregation $(\rho)$ was determined for each colonization incidence according to the relationship depicted in Figure 4. Calculations were made with equation 15 under the assumption that the orchard size $(T)$ was 300,000 trees, the confidence level $(1-\alpha)$ was $90 \%$, and the number of blossoms sampled from each tree $(n)$ was 20. blossom imprints that exhibit bacterial colonies typical of $E$. amylovora.

To test the validity of the sampling procedure, blossoms from two commercial orchards were sampled in 2002. The incidences of blossom colonization in these orchards, as determined with the RD-medium, were 40.5 and $36.6 \%$. For the purpose of validation, these incidences were considered to be the true blossom colonization incidences. According to the sampling procedure, sampling of 20 blossoms from each of four randomly chosen trees would enable the true colonization incidence to be estimated with an accuracy of $10 \%$ in $90 \%$ of the cases. The colonization incidence was calculated for 100 samples of four trees, randomly chosen from the whole sample (i.e., the 25 trees). The four-tree means of the 100 samples had a mean value which was not significantly different from the true colonization incidence of the blossoms (one-tail $t$ test; $P \leq 0.05$ ). Departure of the frequency distribution presented in Figure 10 from normality was tested with the $W$ test of Shapiro and Wilk. The null hypothesis that the frequency distribution differed significantly from a normal distribution was rejected $(W=0.967 ; P[W]=0.079)$. In 88 of the 100 samples, the four-tree means fell within the range of $\pm 10 \%$ from the true blossom colonization incidence (Fig. 10). Analysis of the data obtained in the second orchard gave similar results; the frequency distribution of the sample mean resembled a normal distribution around the true colonization incidence, and $91 \%$ of the four-tree means fell within a range of $\pm 10 \%$ from the true blossom colonization incidence (data not shown).

\section{DISCUSSION}

Reliable identification of bacteria is a complicated task which involves numerous steps (19). Bacteria need to be isolated, purified, and then tested in comparison with reference bacteria isolates. Such comparisons may be based on morphological, gramstaining, biochemical, or molecular criteria. The time required for reliable identification usually is measured in days. The process is labor intensive and, in most cases, the procedure requires skilled personnel and is done in adequately equipped laboratories. When identification is to be conducted by nonskilled personnel, not working in adequately equipped laboratories (such as pear growers in the orchards), the task is even more complicated. In the present study, E. amylovora colonies developed on the RDmedium could be distinguished easily from colonies of other bacteria that commonly inhabit pear blossoms (Fig. 1); false positive and false negative diagnoses were rare (Table 1), and the identi-

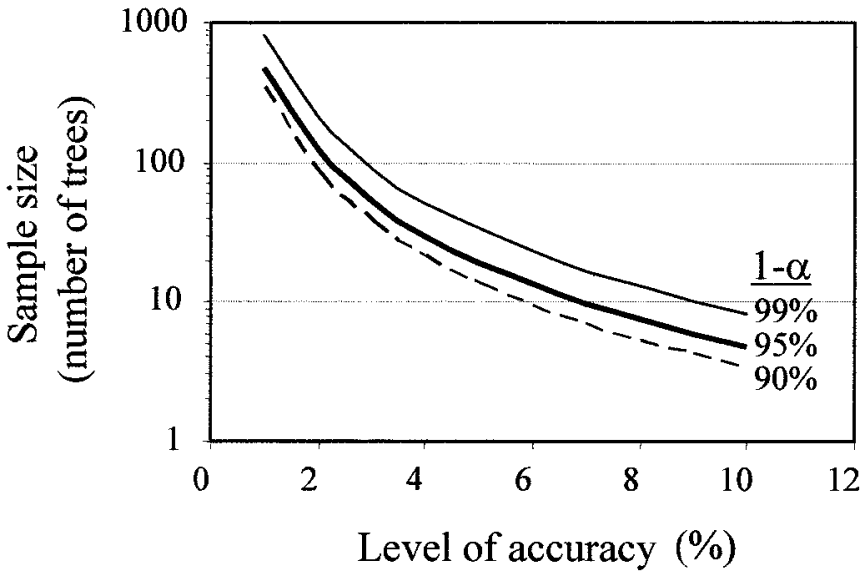

Fig. 9. Number of pear trees to be sampled for optimal estimation of the incidence of blossom colonization by Erwinia amylovora, at chosen levels of accuracy and confidence ( $L$ and $1-\alpha$, respectively). Calculations were made with equation 15 under the assumption that the incidence of blossom colonization $(\pi)$ was $50 \%$, the orchard size $(T)$ was 300,000 trees, and the number of blossoms sampled from each tree $(n)$ was 20. 
fication process was completed within $36 \mathrm{~h}$ (Fig. 2), which is rapid enough for the information gained to be used to guide decision making. It recently was reported that application of oxolinic acid up to $48 \mathrm{~h}$ after inoculating pear blossoms with $E$. amylovora still resulted in fire blight suppression as good as that achieved when the bactericide was applied just before or soon after the time of inoculation (32).

As indicated above, development of a reliable identification tool for determining whether pear blossoms are colonized with E. amylovora is only the first step. Equally important is the development of a blossom-sampling procedure for which a basic requirement is the determination of the spatial distribution of colonized blossoms in the orchard. Results of geostatistical analyses revealed that $E$. amylovora may be spatially distributed both in clusters and at random (Fig. 3), as might have been expected in view of the epidemiology of the pathogen. E. amylovora survives and overwinters in holdover cankers which form a source of initial inoculum. From this source, bacteria are spatially disseminated in the air (by rain splashes or air current) or are transferred by pollinating insects $(15,36,39,40)$. When the number of holdover cankers per unit area is low, or when the spatial dissemination of bacteria in time and space is limited, the likelihood of blossom colonization and infection are higher for blossoms that are adjacent to the sources of initial inoculum than for those that are further away. Accordingly, in these cases, the colonized blossoms are spatially distributed in patches around the holdover cankers. However, when the number of holdover cankers per unit area is large, or when pollinating insects repeatedly transfer bacterial cells from blossom to blossom, then the colonized blossoms are randomly distributed in space. Further analyses of the data enabled quantification of the relationship between the blossom colonization incidence and the coefficient of aggregation (a measure of the spatial distribution). It was found that, when the colonization incidence was below $3 \%$, the pathogen was spatially distributed in clusters, but when the incidence of blossom colonization exceeded $15 \%$, the pathogen distribution was random (Fig. 4). On the basis of these findings, a statistical model was designed and developed to encompass both possible spatial distributions.

The statistical sampling model was employed initially for sensitivity tests. The ultimate conclusion from these tests was that the parameter that determines the necessary sample size is the required level of accuracy $(L)$. Whereas sampling of 20 blossoms from each of several hundred trees was required for estimating the incidence of blossom colonization with an accuracy of $1 \%$, sampling of only a few single trees was enough for an accuracy of $10 \%$ (Figs. 8 and 9). The influence of the other parameters included in the statistical model on the sample size was relatively minor (Figs. 5 to 7). The sampling procedure that was developed was validated with an independent data set and found to be accurate (Fig. 10).

How can the developed sampling procedure improve fire blight management? In our opinion, the sampling procedure should be used in conjunction with a reliable warning system, such as MARYBLYT, BIS95, Cougarblight 98C, or FBCA $(3,31,33,35)$, operated on the basis of a 48 -h weather forecast. When predictions issued by the warning system indicate that E. amylovora infections are not likely to occur, sampling of pear blossoms would not be required. In such situations, even if blossoms are colonized with E. amylovora, the bacteria would continue to develop epiphytically, and would not harm the plants. On the other hand, when the warning system warns that the environmental conditions are likely to promote infections, sampling of blossoms would be required. It can be presumed that the improvement in disease management achieved by blossom sampling would be dependent on the host-pathogen relationships. In situations where fire blight infections do not endanger the survival of the trees (e.g., resistant cultivars, trees that are more than 30 years old, and so on), only the current-year yield is in danger. The number of blossom clusters produced by each tree is much larger than the number of fruit the tree can support; therefore, fruitlets are naturally aborted without any effect on yield. Consequently, it may be possible to develop an action-threshold value based on the blossom colonization incidence. For example, let us assume that the action threshold is set to a colonization incidence of $15 \%$; then, spraying would be justified whenever the blossom colonization incidence (as determined by sampling) was estimated to be greater than the action threshold, plus $L$ (the accuracy level). For example, for an accuracy level of 5\%, spraying would be justified if the estimated blossom colonization incidence was $20 \%$ or more. In such cases, sampling would improve fire blight management by minimizing the risk of a false negative decision (that is, a decision not to apply a spray that was actually needed). Similarly, if the blossom colonization incidence were estimated to be lower than the action threshold value, minus $L$ (i.e., less than 10\%), then spraying would not be needed. In such cases, sampling would improve fire blight management by minimizing the risk of a false positive decision (i.e., a decision to apply a spray that was actually not needed). If the estimated incidence of blossom colonization fell within the range of the action threshold $\pm L$ (i.e., between 10 and $20 \%$ ), the decision maker would need to reach his or her own decision about the action to be taken. Presumably, the decisions would reflect that person's risk perception: individuals who do not tolerate risks would probably prefer to spray, whereas those who are willing to take risks would not.

The contribution of sampling to fire blight management would be different in situations where fire blight infections might ultimately kill the trees. Potentially, even a few single infections could have devastating consequences; therefore, the action threshold value should be set to a colonization incidence of $0 \%$. In that case, the contribution of sampling to fire blight management would be dependent on the result of the sampling. If the estimated incidence of blossom colonization was greater than $0 \%$, it would mean that inoculum was present and spraying would be justified. Consequently, in these cases, sampling would reduce the risk of a false negative management decision. However, if the incidence of blossom colonization was estimated to be $0 \%$, then it would not be possible to distinguish between situations where the pathogen

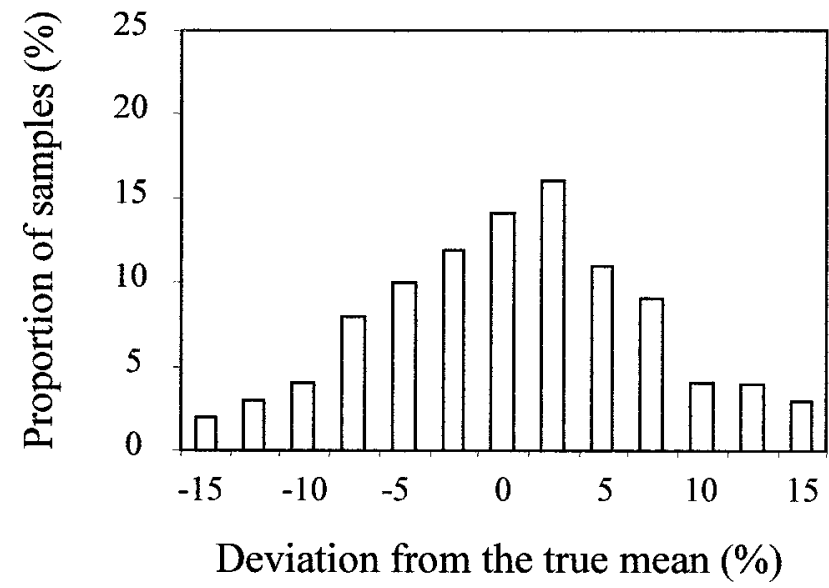

Fig. 10. Evaluation of the procedure for sampling pear blossom with an independent data set. Twenty pear blossoms were sampled from each of 25 pear trees in a commercial orchard that was not treated with bactericides, in 2002. The Erwinia amylovora colonization incidence was determined with the rapid diagnosis medium. The mean colonization incidence of the blossoms in the 25 trees, $40.5 \%$, was considered as the true colonization incidence. Based on the sampling procedure, sampling of 20 blossoms from each of four trees would enable the true colonization incidence to be estimated with an accuracy of $\pm 10 \%$ at a confidence level of $90 \%$. One hundred samples of four trees were randomly chosen from the data set, and the mean colonization incidence was calculated for each sample. The bars represent the frequency distribution of deviations from the true colonization incidence for the 100 random samples of four trees. 
is really absent and those in which it is present at incidences smaller than the level of accuracy used. In such cases, sampling would not assist the decision maker in his or her deliberations. The situation could be improved by implementing a high level of accuracy (e.g., $1 \%$ instead of 5 or $10 \%$ ). However, the problem then is that obtaining such a high level of accuracy requires a very large sample size (Fig. 9); it might be impracticable to sample 20 blossoms from several hundred trees before each decision. The conclusion that emerges from this discussion is that sampling of pear blossoms and estimation of the incidence of blossom colonization by E. amylovora could improve fire blight management, but not in all cases.

\section{ACKNOWLEDGMENTS}

This research was supported in part by the Chief Scientist of the Israeli Ministry of Agriculture and by the Israeli Council of Fruit Trees. Contribution no. 514/02 from the Agricultural Research Organization, the Volcani Center, Bet Dagan 50250, Israel. We thank Y. Rekah for performing the geostatistical analyses.

\section{LITERATURE CITED}

1. Bereswill, S., Jock, S., Bellemann, P., and Geider, K. 1998. Identification of Erwinia amylovora by growth morphology on agar containing copper sulfate and by capsule staining with lectin. Plant Dis. 82:158-164.

2. Bereswill, S., Pahl, A., Bellemann, P., Zeller, W., and Geider, K. 1992. Sensitive and species-specific detection of Erwinia amylovora by polymerase chain reaction. Appl. Environ. Microbiol. 58:3522-3526.

3. Billing, E. 1980. Fire blight (Erwinia amylovora) and weather: A comparison of warning systems. Ann. Appl. Biol. 95:365-377.

4. Bochner, B. R. 1989. Sleuthingout bacterial identities. Nature 339:157158.

5. Bochner, B. R. 1989. Breathprints at the microbial level. ASM News 55:536-539.

6. Brulez, W., and Zeller, W. 1981. Seasonal changes of epiphytic Erwinia amylovora on ornamentals in relation to weather conditions and course of infections. Acta Hortic. 117:37-43.

7. Chellemi, D. O., Rohrbach, K. G., Yost, R. S., and Sonoda, R. M. 1998. Analysis of the spatial pattern of plant pathogens and disease plants using geostatistics. Phytopathology 78:221-226.

8. Collett, D.1991. Modelling Binary Data. 2nd ed. Chapman \& Hall, London.

9. Crosse, J. E., and Goodman, R. N. 1973. A selective medium for a definitive colony characteristic of Erwinia amylovora. Phytopathology 63:1425-1426.

10. Gaunt, R. E., and Cole, M. J. 1992. Sequential sampling for wheat stripe rust management. Crop Prot. 11:138-140.

11. Hughes, G., Madden L. V., and Munkvold, G. P. 1996. Cluster sampling for disease incidence data. Phytopathology 86:132-137.

12. Hughes, G., McRoberto, N., Madden, L. V., and Gottward, T. R. 1997. Relationships between disease incidence at two levels in a spatial hierarchy. Phytopathology 87:542-550.

13. Isaaks, E. H., and Srivastava, R. M. 1989. Applied Geostatistics. Oxford University Press, New York.

14. Ishimau, C., and Klos, E. J. 1984. New medium for detecting Erwinia amylovora and its use in epidemiological studies. Phytopathology 74:1342-1345.

15. Johnson, K. B., and Stockwell, V. O. 1998. Management of fire blight: A case study in microbial ecology. Annu. Rev. Phytopathol. 36:227248.

16. Jones, J. B., Chase, A. R., and Harris, G. K. 1993. Evaluation of the Biolog GN microplate system for identification of some plant-pathogenic bacteria. Plant Dis. 77:553-558.
17. Journal, A. G., and Huijbregts, C. J. 1978. Mining Geostatistics. Academic Press, New York.

18. Kado, C. I., and Heskett, M. G. 1970. Selective media for isolation of Agrobacterium, Corynebacterium, Erwinia, Pseudomonas, and Xanthomonas. Phytopathology 60:969-976.

19. Krieg, N. R., and Holt, J. G. 1984. Bergey's Manual of Systematic Bacteriology, Vols. 1 and 2. Williams \& Wilkins, London.

20. Larkin, R. P., Gumpertz, M. L., and Ristaino, J. B. 1985. Geostatistical analysis of Phytophthora epidemics development in commercial bell pepper fields. Phytopathology 85:191-203.

21. Lelliot, R. A. 1967. The diagnosis of fire blight (Erwinia amylovora) and some diseases caused by Pseudomonas syringae. Eur. Mediterr. Plant Prot. Org. Publ. Ser. A(45-E):27-34.

22. Madden, L. V., and Hughes, G. 1995. Plant disease incidence: Distributions, heterogeneity, and temporal analysis. Annu. Rev. Phytopathol. 33:529-564.

23. Madden, L. V., and Hughes, G. 1999. Sampling for plant disease incidence. Phytopathology 89:1088-1103.

24. Madden, L. V., Hughes, G., and Ellis, M. A. 1995. Spatial heterogeneity of the incidence of grape downy mildew. Phytopathology 85:269-275.

25. Madden, L. V., Hughes, G., and Munkvold, G. P. 1996. Plant disease incidence: Inverse sampling, sequential sampling and confidence intervals when observed mean incidence is zero. Crop Prot. 15:621-632.

26. Miller, T. D., and Schroth, M. N. 1972. Monitoring the epiphytic population of Erwinia amylovora on pear with a selective medium. Phytopathology 62:1175-1182.

27. Parker, S. R., Shaw, M. W., and Royle, D. J. 1997. Measurements of spatial patterns of disease in winter wheat crops and the implications for sampling. Plant Pathol. 46:470-480.

28. Rekah, Y., Shtienberg, D., and Katan, J. 1999. Spatial distribution and temporal development of Fusarium crown and root rot of tomato and pathogen dissemination in field soil. Phytopathology 89:831-839.

29. Sasser, M. 1990. Identification of bacteria through fatty acid analysis. Pages 199-204 in: Methods in Phytobacteriology. Z. Klement, K. Rudolf, and D. Sand, eds. Akademiai Kiado, Budapest, Hungary.

30. Sholberg, P. L., Bedford, K. E., Haag, P., and Randall, P. 2001. Survey of Erwinia amylovora isolates from British Colombia for resistance to bactericides and virulence on apple. Can. J. Plant Pathol. 23:60-67.

31. Shtienberg, D., Kritzman, G., Herzog, Z., Oppenheim, D., Zilberstaine, M., and Blatchinsky, D. 1999. Development and evaluation of a decision support system for management of fire blight in pears. Acta Hortic. 489:385-392.

32. Shtienberg, D., Zilberstaine, M., Oppenheim, D., Herzog, Z., Manulis, S., Shwartz, H., and Kritzman, G. 2001. Efficacy of oxolinic acid and other bactericides in suppression of Erwinia amylovora in pear orchards in Israel. Phytoparasitica 29:143-154.

33. Smith, T. J. 1993. A predictive model for forecasting fire blight of pear and apples in Washington State. Acta Hortic. 338:153-160.

34. Stein, A., Kocks, C. G., Zadoks, J. C., Frinking, H. D., Ruissen, M. A., and Myers, D. E. 1994. A geostatistical analysis of the spatio-temporal development of downy mildew epidemics in cabbage. Phytopathology 84:1227-1239.

35. Steiner, P. W. 1990. Predicting apple blossom infections by Erwinia amylovora using the MARYBLYT model. Acta Hortic. 273:139-148.

36. Thomson, S. V. 2000. Epidemiology of fire blight. Pages 9-36 in: Fire Blight: The Disease and Its Causative Agent, Erwinia amylovora. J. L. Vanneste, ed. CABI Publishing, Wallingford, UK.

37. Turechek, W. W., Ellis, M. A., and Madden, L. V. 2001. Sequential sampling for incidence of Phomopsis leaf blight of strawberry. Phytopathology 91:336-347.

38. Turechek, W. W., and Madden, L. V. 1999. Spatial pattern analysis and sequential sampling for the incidence of leaf spot on strawberry in Ohio. Plant Dis. 83:992-1000.

39. van der Zwet, T. 1996. Present world-wide distribution of fire blight. Acta Hortic. 411:7-8.

40. van der Zwet, T., and Beer, S. V. 1995. Fire blight-Its nature, prevention, and control. U.S. Dep. Agric. Bull. No. 631. 